\title{
O consumo de produto à base de yacon (Smallanthus sonchifolius) aumenta glucagon-like peptide-1 e diminui o peso corporal de ratas Wistar ovariectomizadas
}

\section{Yacon-based product consumption (Smallanthus sonchifolius) increases glucagon-like peptide-1 and reduces the body weight of ovariectomized Wistar rats}

\author{
Joice de Fátima Laureano Martins da Silva ${ }^{1}$, Kamilla Milione Nogueira Reis ${ }^{2}$, \\ Mariane Laureano Florindo de Freitas ${ }^{3}$, Viviane da Cruz Rafael ${ }^{4}$, France \\ Coelho Araújo ${ }^{5}$, Sirlene de Souza Rodrigues Sartori ${ }^{6}$, Edimar Aparecida \\ Filomeno Fontes ${ }^{7}$, Célia Lúcia de Luces Fortes Ferreira ${ }^{8}$
}

\section{Resumo}

\begin{abstract}
A raiz do yacon (Smallanthus sonchifolius), em função da rica concentração de fruto-oligossacarídeos, é classificada como prebiótico e tornou-se promissora da obesidade pelo aumento da saciedade. O objetivo deste estudo foi avaliar a inclusão de um produto à base de yacon (PBY) em ratas ovariectomizadas (OVX) no consumo alimentar, na modulação de medidas antropométricas e do imunomarcador da saciedade glucagon-like peptide-1 (GLP-1) em ratas Wistar ovariectomizadas (OVX). Analisou-se o consumo alimentar pela pesagem diária de sobra de dieta, a porcentagem de gordura corporal foi determinada pelo índice de Lee e também foram avaliados o peso, o índice de massa corporal (IMC) e circunferência abdominal. Fragmentos do ceco foram utilizados para imunomarcação de GLP-1, de ratas OVX, após serem alimentadas por 24 semanas com dieta padrão adicionadas ou não de $6 \%$ de FOS/inulina/PBY. Observou-se diminuição da circunferência abdominal ( $\mathrm{p}=0,2173)$ em 3,5\%, também houve decréscimo de IMC ( $\mathrm{p}=0,3822)$ em $6,25 \%$ e de percentual de gordura corporal $(\mathrm{p}=0,3528)$ em $2,14 \%$ em animais que receberam PBY durante 24 semanas (G4) comparado aos animais do grupo controle. No grupo G4 o GLP-1 aumentou $(\mathrm{p}<.0001)$, os animais aumentaram o consumo $(\mathrm{p}=0,0064)$ e, paradoxalmente, tiveram menor ganho de peso $(\mathrm{p}<.0001)$, o que pode estar associado ao fato de que as fibras diminuem a eficiência de absorção de lipídeos ao longo do intestino delgado, o que pode diminuir a assimilação calórica de nutrientes. Esse fenômeno demonstra que o PBY possui potencial na modulação da obesidade, portanto, melhoria da qualidade de vida de mulheres na menopausa.
\end{abstract}

Palavras-chave: Yacon. Avaliação antropométrica. Consumo alimentar. Glucagon-like peptide-1.

\footnotetext{
${ }^{1}$ Doutorado em Ciência e Tecnologia de Alimentos pela Universidade Federal de Viçosa (UFV), Viçosa, Minas Gerais, Brasil. E-mail: martinsjil@yahoo.com.br

${ }^{2}$ Graduação em Enfermagem pela Universidade Federal de São João del-Rei (UFSJ), São João del-Rei, Minas Gerais, Brasil.

${ }^{3}$ Mestranda em Ciência e Tecnologia de Alimentos no Instituto Federal de Educação, Ciência e Tecnologia do Sudeste de Minas Gerais (IF Sudeste MG), Rio Pomba, Minas Gerais, Brasil.

${ }^{4}$ Doutorado em Ciência e Tecnologia de Alimentos pela Universidade Federal de Viçosa, Viçosa, Minas Gerais, Brasil.

${ }^{5}$ Mestrado em Ciência da Nutrição (Saúde Pública) pela Universidade Federal de Viçosa, Viçosa, Minas Gerais, Brasil. Docente do Curso de Medicina e Diretora da Faculdade de Enfermagem do Centro Universitário Governador Ozanam Coelho (UniFagoc), Ubá, Minas Gerais, Brasil.

${ }^{6}$ Doutorado em Biologia Celular e Estrutural pela Universidade Federal de Viçosa, Viçosa, Minas Gerais, Brasil.

${ }^{7}$ Doutorado em Ciência e Tecnologia de Alimentos pela Universidade Federal de Viçosa, Viçosa, Minas Gerais, Brasil. Docente do Departamento de Tecnologia de Alimentos da Universidade Federal de Viçosa, Viçosa, Minas Gerais, Brasil.

${ }^{8}$ Doutorado em Ciência de Alimentos pela Oklahoma State University (OSU), Oklahoma, Estados Unidos da América do Norte. Professora Titular da Universidade Federal de Viçosa, Centro de Ciências Exatas e Tecnológicas, Departamento de Tecnologia de Alimentos, Viçosa, Minas Gerais, Brasil.
} 


\begin{abstract}
The yacon root (Smallanthus sonchifolius), due to the rich concentration of fructo-oligosaccharides, is classified as prebiotic and has become promising for obesity due to increased satiety. The aim of this study was to evaluate the inclusion of a yacon-based product (PBY) in ovariectomized rats (OVX) in food consumption, in the modulation of anthropometric measurements and in the satiety immunosorbent glucagon-like peptide-1 (GLP-1) in rats Wistar ovariectomized (OVX). Food consumption was analyzed by daily weighing of leftover diet, the percentage of body fat was determined by the Lee index, and weight, body mass index (BMI) and waist circumference were also evaluated. Cecum fragments were used for immunostaining GLP-1, from OVX rats, after being fed for 24 weeks with a standard diet with or without $6 \%$ FOS / inulin / PBY. There was a decrease in abdominal circumference $(p=0.2173)$ in $3.5 \%$, there was also a decrease in BMI $(\mathrm{p}=0.3822)$ in $6.25 \%$ and a percentage of body fat $(\mathrm{p}=0.3528) 2.14 \%$ in animals that received PBY for 24 weeks (G4) compared to animals in the control group. In the G4 group GLP-1 increased $(p<.0001)$, the animals increased their consumption $(p=0.0064)$ and paradoxically, they gained less weight gain $(\mathrm{p}<.0001)$, which may be associated with the fact that fibers decrease the efficiency of absorption of lipids along the small intestine, which can decrease the caloric assimilation of nutrients. This phenomenon demonstrates that PBY has the potential to modulate obesity, thus improving the quality of life of women in menopause.
\end{abstract}

Keywords: Yacon. Anthropometric assessment. Food consumption. Glucagon-like peptide-1.

\section{Introdução}

A crescente preocupação da população com a saúde tem resultado numa maior demanda por alimentos funcionais, assim como no aumento de pesquisas com o intuito de elucidar os benefícios proporcionados pelas substâncias bioativas presentes nesses alimentos, ${ }^{(1)}$ sendo esses usados como parte de uma dieta normal que proporciona benefícios fisiológicos e/ou reduz o risco de doenças crônicas não transmissíveis, além de suas funções nutricionais básicas. ${ }^{(2)}$

A yacon (Smallanthus sonchifolius) é uma planta nativa da Cordilheira dos Andes e distribuise naturalmente desde Colômbia e Venezuela até o noroeste da Argentina. ${ }^{(2)}$ Essa planta foi introduzida no Brasil por descendentes japoneses por volta de 1989; era cultivada em altitudes variadas, como entre $900 \mathrm{~m}$ e $3.500 \mathrm{~m}$ acima do nível do mar na Bolívia, Equador e Peru; a 600 m e 2.500 m é cultivada na Argentina; já no Brasil está entre $600 \mathrm{~m}$ e $800 \mathrm{~m}$, e ao nível do mar é cultivada no Japão e na Nova Zelândia. ${ }^{(3)} \mathrm{O}$ modo de consumo da yacon pode variar de acordo com sua utilização; nos mercados andinos a yacon é classificada como fruta, devido ao seu sabor adocicado e refrescante, onde é exposta juntamente com outras frutas e não com batatas, tubérculos e raízes. ${ }^{(3)}$

Dentre os alimentos funcionais, a raiz do yacon tem se destacado pelo seu alto teor de frutanos, nomeadamente fruto-oligossacarídeos (FOS) e inulina, o que lhe confere propriedades funcionais diversas, ${ }^{(2)}$ incluindo a modulação da obesidade. $^{(3)}$

Considerada um grande problema de saúde pública, a obesidade apresenta-se como sendo uma doença de origem multifatorial, que afeta e traz implicações para uma significativa parcela da população mundial. Além disso, representa um dos maiores fatores de risco para doenças cardiovasculares e diabetes. $^{(2)}$

A obesidade é uma doença crônica caracterizada pelo excesso de tecido adiposo no organismo, ${ }^{(4)}$ sendo uma comorbidade comum em mulheres principalmente no período da menopausa, em função do hipoestrogenismo. ${ }^{(5)}$ Além do característico acúmulo progressivo da gordura abdominal, o estabelecimento da obesidade está relacionado ao aumento de comorbidades relacionadas, quais sejam: diabetes mellitus tipo 2 (DM2), hipertensão arterial sistêmica (HAS), ${ }^{(6)}$ osteoartrite, apneia do sono, dislipidemia e câncer. ${ }^{(7)}$ 
A raiz do yacon é um alimento altamente perecível, e para contornar essa característica vislumbrou-se a elaboração de um produto que garantisse acesso a essa rica fonte de FOS/inulina durante todo o ano; assim, a raiz foi processada gerando um produto à base de yacon (PBY) com maior vida de prateleira e elevado teor de FOS/ inulina (19 g/100 g do produto). ${ }^{(8)} \mathrm{O}$ PBY mostrouse efetivo na saúde óssea, ${ }^{(3)}$ na constipação intestinal $(\mathrm{CI})^{(2)}$ e na modulação no índice glicêmico. ${ }^{(2)}$ No presente estudo avaliou-se o papel do produto elaborado a partir da raiz do yacon processada (PBY), fonte de FOS/inulina, na modulação de alterações antropométricas decorrentes da menopausa, bem como ação no consumo alimentar, e no glucagon-like peptide-1 (GLP-1), marcador da saciedade.

\section{Material e Métodos}

A experimentação foi desenvolvida no Laboratório de Frutas e Hortaliças no Departamento de Tecnologia de Alimentos, Laboratório de Nutrição Experimental no Departamento de Nutrição e Saúde e no Laboratório de Tecnologia e Propriedades da Madeira, da Universidade Federal de Viçosa (UFV), Minas Gerais. O projeto foi submetido ao Comitê de Ética na Pesquisa com Animais - UFV; os procedimentos experimentais foram aprovados pela Comissão de Ética do Departamento de Medicina Veterinária da Universidade Federal de Viçosa, Minas Gerais (Parecer n ${ }^{\circ}$ 55/2011).

Composição química do produto

à base de yacon (PBY)

As análises de composição centesimal do PBY foram conduzidas conforme protocolos oficiais de umidade, determinada em estufa a $105^{\circ} \mathrm{C}$ até peso constante; para o teor de proteínas foi utilizado o método de Kjeldahl; ${ }^{(9)}$ lipídeo, pelo método de Soxhlet; ${ }^{(9)}$ e cinzas, por processo gravimétrico, utilizando-se a carbonização das amostras, seguida de incineração em mufla a $550^{\circ} \mathrm{C}$ até peso constante.
O teor de fibra alimentar solúvel (FAS) e fibra alimentar insolúvel (FAI) foram quantificados de acordo com o método enzimático-gravimétrico utilizando-se o kit enzimático específico. ${ }^{(9)}$

Oligofrutanos (FOS e inulina) foram determinados por meio de cromatografia líquida de alta eficiência (CLAE), ${ }^{(10)}$ utilizando coluna BIORAD HPX 87P (fase estacionária de chumbo), e água purificada como fase móvel. As amostras foram diluídas (1 g em $100 \mathrm{~mL}$ de água destilada), centrifugadas a $11.269 \times \mathrm{g}$, e, em seguida, filtradas através de uma membrana de decafluoreto (PVDF) de polivinil Millipore com $0,22 \mu \mathrm{m}$ de porosidade e $13 \mathrm{~mm}$ de diâmetro. As amostras foram injetadas em CLAE (Varian, ProStar 410 HPLC Auto Sampler; Varian Inc, USA) com detector de índice de refração e injetor automático, com as seguintes condições de operação: taxa de fluxo de $0,6 \mathrm{~mL}$. min-1 e temperatura de coluna $85^{\circ} \mathrm{C}$.

Medição de calorimetria (poder calorífico) da dieta

Amostras das dietas foram submetidas à secagem para retirada da umidade a $70{ }^{\circ} \mathrm{C}$ em estufa (Fanem ${ }^{\circledR}$, modelo 315SE, São Paulo) por vinte e quatro horas, seguindo-se a maceração e peneiradas. A fração para análise foi a que ultrapassou a peneira de MESH/TYLER 40, e a fração (resíduo) que ficou retida na peneira de MESH/TYLER 60 foi descartada. Após esse procedimento, a amostra foi submetida ao Calorímetro Adiabático C-5000 $\left(\mathrm{IKA}^{\circledR}\right.$, São Paulo) para leitura do poder calorífico.

Origem e manutenção dos animais

Ao todo, foram utilizadas 20 ratas (Rattus norvegicus, variedade albinus, classe Rodentia), recém-desmamadas (28 dias), da linhagem Wistar, provenientes do Biotério Central do Centro de Ciências Biológicas e da Saúde da Universidade Federal de Viçosa. Foram mantidas no Laboratório de Nutrição experimental em gaiolas individuais de aço inoxidável a $22 \pm 2{ }^{\circ} \mathrm{C}$ e luzes controladas (fotoperíodo de doze horas) e receberam água 
ad libitum. Após, os animais foram dispostos em diferentes grupos experimentais e receberam as dietas purificadas e manipuladas, acrescidas de quantidades de PBY suficiente para fornecer uma dose de $6 \%$ de FOS/inulina. Na primeira fase do estudo (12 semanas) os animais receberam dieta purificada manipulada (AIN-93G) - (Tabela 1), e na segunda fase (20 semanas de vida) foi administrada a dieta purificada manipulada (AIN-93M ) (Tabela 2). ${ }^{(10)}$

Tabela 1 - Composição das dietas administradas na fase de crescimento (12 semanas).

\begin{tabular}{lcc}
\hline Ingredientes & $\begin{array}{c}\text { Dieta controle } \\
(\mathbf{\%})\end{array}$ & $\begin{array}{c}\text { Dieta teste (suplementada } \\
\text { com 6\% de FOS) (\%) }\end{array}$ \\
\hline Caseína & 20,00 & 20,00 \\
Amido dextrinizado & 13,20 & - \\
Sacarose & 10,00 & - \\
Óleo de soja & 7,00 & 7,00 \\
Fibra (celulose microfina) & 5,00 & 3,02 \\
Mistura de minerais (AIN-93G) & 3,50 & 3,50 \\
Mistura de vitaminas & 1,00 & 1,00 \\
L-cistina & 0,3 & 0,30 \\
Bitartarato de colina & 0,25 & 0,25 \\
Amido de milho & 39,75 & - \\
Produto à base de yacon (PBY) & - & 64,93 \\
\hline Total & 100,00 & 100,00 \\
\hline
\end{tabular}

Fonte: Adaptado de Reeves et al..$^{(10)}$

Legenda: Composição da raiz do yacon processada (PBY) (g/100 g) de FOS/inulina: 9,24\%; sacarose: 10,08\%; frutose: $12,60 \%$; glicose: 9,40\%; proteína: $0,32 \%$; umidade: $60,66 \%$; cinzas: $0,51 \%$; lipídios: $0 \%$. Os teores de açúcares livres presentes no PBY adicionado à formulação foram descontados da sacarose, maltodextrina e amido de milho, para manutenção da proporção preconizada pela AIN-93G.

Tabela 2 - Composição das dietas administradas na fase de manutenção (8 semanas).

\begin{tabular}{lcc}
\hline Ingredientes & $\begin{array}{c}\text { Dieta controle } \\
(\mathbf{\%})\end{array}$ & $\begin{array}{c}\text { Dieta teste (suplementada } \\
\text { com 6\% de FOS) (\%) }\end{array}$ \\
\hline Caseína & 14,00 & 14,00 \\
Amido dextrinizado & 15,50 & - \\
Sacarose & 10,00 & - \\
Óleo de soja & 4,00 & 4,00 \\
Fibra (celulose microfina) & 5,00 & 5,00 \\
Mistura de minerais (AIN-93G) & 3,50 & 3,50 \\
Mistura de vitaminas & 1,00 & 1,00
\end{tabular}




\section{Continuação}

L-cistina

Bitartarato de colina

Amido de milho

46,57

39,68

Produto à base de yacon (PBY)

32,39

Total

100,00

100,00

Fonte: Adaptado de Reeves et al. ${ }^{(10)}$

Legenda: Composição da raiz do yacon processada (PBY) (g/100 g) de FOS/inulina: 18,52\%; sacarose: 13,19\%; glicose: 6,09\%; proteína: 1,51\%; umidade: 55,11\%; cinzas: 4,72\%; lipídios: 0\%. Os teores de açúcares livres presentes no PBY adicionado à formulação foram descontados da sacarose, maltodextrina e amido de milho, para manutenção da proporção preconizada pela AIN-93M.

\section{Delineamento experimental}

O estudo foi realizado em conformidade com

o delineamento experimental abaixo.

Figura 1 - Organograma do experimento.

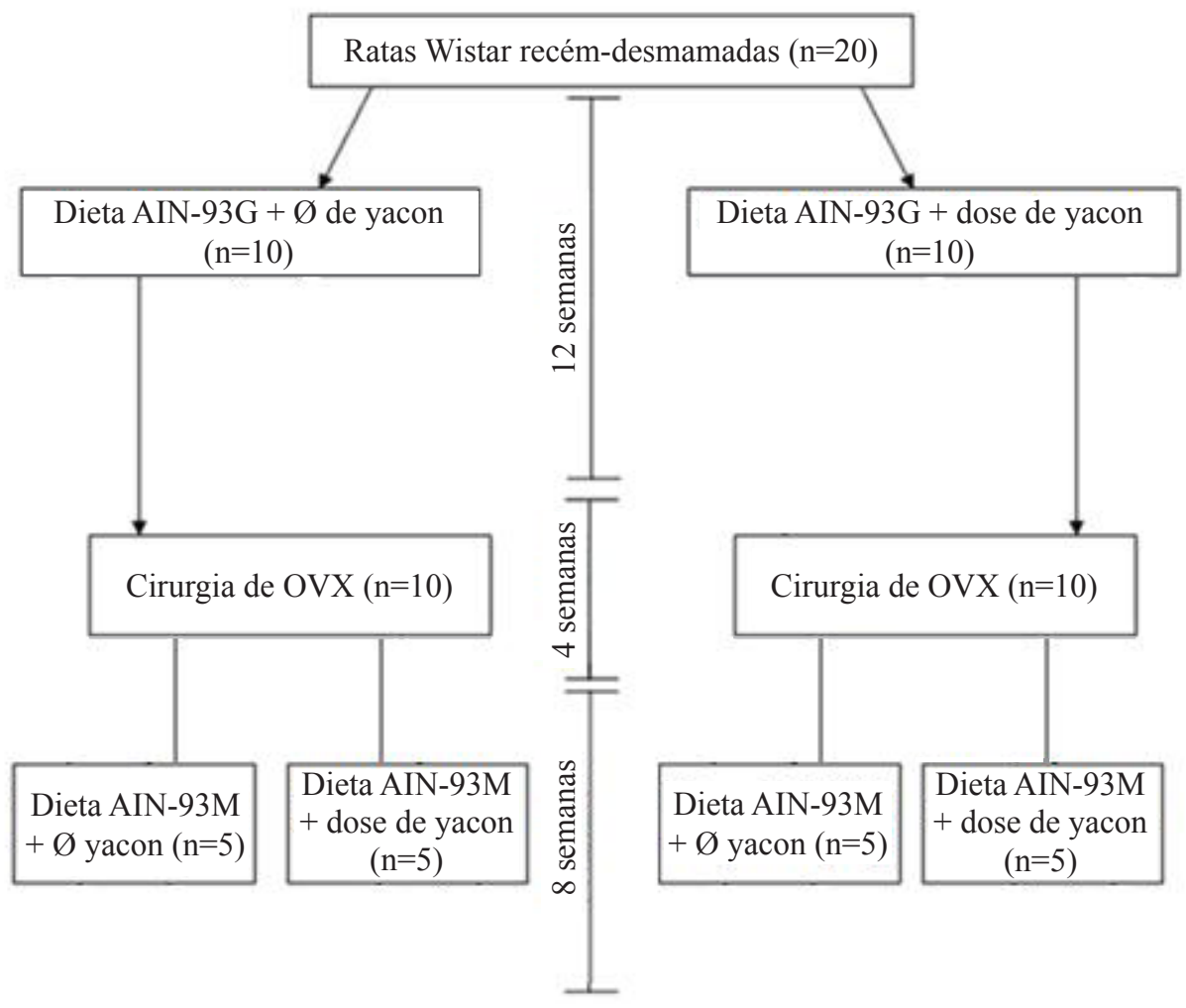

Fonte: Autoras

As ratas foram divididas em dois grupos por delineamento inteiramente casualizado (DIC), sendo um grupo teste $(\mathrm{n}=10)$, um grupo controle $(\mathrm{n}=10)$ e amostra $(\mathrm{n}=20)$, e foram acompanhadas até completarem 24 semanas de vida. Durante 12 semanas foi administrada aos animais a dieta 
de crescimento AIN-93G, com e sem PBY; após esse período, os animais foram submetidos ao procedimento cirúrgico para retirada de ovário (ovariectomia - OVX). Como os resultados da cirurgia não influenciaram nos resultados, os dados não foram utilizados nos resultados e discussão. $\mathrm{Na}$ sequência, houve um washout de quatro semanas para recuperação. Após a recuperação, os animais foram alocados em quatro grupos $(n=5)$, conforme a Figura 1. Ao final do experimento, os animais foram eutanasiados por uma overdose de dióxido de carbono ${ }^{(11)}$ para realização das análises subsequentes.

\section{Ovariectomia ou laparotomia}

Ao atingirem a fase adulta (12 semanas) os animais foram submetidos à ovariectomia bilateral (OVX). Para o procedimento cirúrgico, trinta minutos prévios os animais receberam o anti-inflamatório flunixina meglumina $(0,68 \mathrm{mg} /$ $\mathrm{kg}$ ) e o antibiótico enrofloxocina $(10 \mathrm{mg} / \mathrm{kg})$, ambos por via subcutânea. Em seguida, receberem associação de diazepan $(2,5 \mathrm{mg} / \mathrm{kg})$ e fentanil $(0,03 \mathrm{mg} / \mathrm{kg})$, por via intraperitoneal. A indução e a manutenção da anestesia foram realizadas com a administração de isofluorano diluído em $100 \%$ de oxigênio, por via inalatória, por meio de vaporizador calibrado. A concentração do isofluorano foi ajustada de forma a manter o plano anestésico adequado. As ratas em decúbito dorsal foram submetidas à depilação da região abdominal lateral e a assepsia local foi realizada com álcool iodado. Em seguida, a pele e a musculatura foram incisadas longitudinalmente na linha medial próxima ao nível dos rins, abaixo da última costela, onde o ovário foi identificado e exposto. Foi realizada a hemostasia através da ligação da parte superior da trompa com um fio, e então foi realizada a excisão ovariana com posterior sutura da musculatura e da pele. ${ }^{(12)}$

Nas primeiras cinco horas após as cirurgias, os animais permaneceram em câmara aquecida, com a finalidade de manter a temperatura corporal. Sequencialmente, procedeu-se a um washout para recuperação, com suspensão dos tratamentos por quatro semanas. Seguiu-se administração da dieta AIN-93M, ${ }^{(10)}$ acrescida ou não de $6 \%$ de FOS/ inulina (de PBY) durante oito semanas, conforme organograma indicado na Figura 1.

Avaliação do tecido adiposo visceral (TAV) e avaliações murinométricas entre os grupos

Os pesos dos animais e a ingestão alimentar foram monitorados diariamente. $\mathrm{O}$ ganho de peso corporal foi determinado pela diferença entre os pesos no dia da eutanásia e o do primeiro dia do experimento. Foi calculado o ganho de peso e o coeficiente de eficiência alimentar (CEA) por percentagem, de acordo com a fórmula: $\mathrm{CEA} \%=$ ganho de peso (g) / consumo alimentar (g) x 100. $\mathrm{O}$ índice de massa corporal (IMC) foi determinado de acordo com Novelli et al. ${ }^{(13)}$ sendo calculado segundo a fórmula: $\mathrm{IMC}=$ peso $(\mathrm{g}) /$ comprimento $(\mathrm{cm})^{2}$, em que o comprimento é a medida nasoanal.

Para avaliação da gordura corporal (GC) utilizou-se o índice de Lee, ${ }^{(14)}$ segundo a fórmula: [peso corporal $(\mathrm{g})]^{1 / 3} /$ comprimento nasoanal $(\mathrm{cm})$.

Realizou-se a avaliação da circunferência abdominal. ${ }^{(13)}$

Imunomarcação de glucagon-like peptide-1 (GLP-1)

A determinação do GLP-1 foi realizada de acordo com protocolos em uso no Laboratório de Histologia no Departamento de Biologia Estrutural da UFV, pela técnica imuno-histoquímica (IHQ) da peroxidase-anti-peroxidase (PAP), ${ }^{(15)}$ utilizando anticorpo monoclonal de coelho anti-GLP-1.

Para revelação, os cortes foram cobertos com solução de cromógeno 3,3' diaminobenzidina (DAB líquido), e para contracoloração os cortes foram imersos em hematoxilina de Harris diluída por 20 segundos. Subsequentemente, as lâminas foram lavadas, desidratadas e montadas em resina Entellan ${ }^{\circledR}$ (Merck, Alemanha). ${ }^{(16)}$

As imagens histológicas foram obtidas por meio de microscópio de luz Olympus AX 70, 
equipado com acessório fotográfico U-PHOTO (Olympus), acoplado a um sistema computadorizado (programa SPOT, versão 3.5.9 para Windows). No total, foram obtidas 1200 imagens (dimensões 1596 x 1196 pxls) para contagens de células de GLP-1 imunomarcadas.

\section{Análise estatística}

Os dados foram analisados no programa estatístico Statistical Analysis System (SAS) versão 9.2, licenciado pela Universidade Federal de Viçosa (UFV), em que a análise de variância foi realizada utilizando o teste $\mathrm{F}$, e para comparação entre as médias dos tratamentos foi utilizado o teste de Duncan a nível de 5\% de probabilidade.

\section{Resultados}

No presente estudo, o PBY foi produzido em duas bateladas, na primeira foi encontrado um teor de 9,24 g 100g-1 de FOS/inulina, e na segunda 18,52 g 100g-1 de FOS/inulina. Os resultados de ganho de peso total, contagem de GLP-1, consumo alimentar total e coeficiente de eficiência alimentar estão dispostos a seguir.

Tabela 3 - Valores médios ( $n=5)$ de ganho de peso (GP), contagem de glucagon-like peptide-1 (GLP-1), consumo alimentar (CA) e coeficiente de eficiência alimentar (CEA), após ovariectomia. (OVX), em ratas alimentadas com dieta controle e dieta teste durante 24 semanas.

\begin{tabular}{|c|c|c|c|c|c|}
\hline \multirow{3}{*}{ Variáveis } & \multicolumn{4}{|c|}{ Grupos } & \multirow{3}{*}{ P valor } \\
\hline & G1 & G2 & G3 & G4 & \\
\hline & \multicolumn{4}{|c|}{ Média/DP } & \\
\hline GP (gramas) & $238,60 \pm 18,73^{\mathrm{a}}$ & $230,60 \pm 10,61^{\mathrm{a}}$ & $244,40 \pm 15,16^{\mathrm{a}}$ & $181,20 \pm 14,84^{b}$ & $<.0001$ \\
\hline GLP-1 (média/campo) & $0,87 \pm 0,24^{\mathrm{a}}$ & $2,94 \pm 0,31^{\mathrm{b}}$ & $1,14 \pm 0,63^{\mathrm{a}}$ & $3,53 \pm 0,06^{\mathrm{c}}$ & $<.0001$ \\
\hline CA (gramas) & $387,22 \pm 24,63^{b}$ & $414,40 \pm 16,93^{\mathrm{a}, \mathrm{b}}$ & $451,76 \pm 27,70^{\mathrm{a}}$ & $444,72 \pm 26,76^{\mathrm{a}}$ & 0.0064 \\
\hline CEA $(\%)$ & $0,62 \pm 0,03^{\mathrm{a}}$ & $0,56 \pm 0,01^{\mathrm{b}}$ & $0,54 \pm 0,04^{b}$ & $0,41 \pm 0,05^{\mathrm{c}}$ & $<.0001$ \\
\hline
\end{tabular}

Fonte: Autoras

Legenda: Médias seguidas de letras iguais, de ganho de peso (GP), contagem de glucagon-like peptide-1 (GLP-1), consumo alimentar (CA) e coeficiente de eficiência alimentar (CEA), na linha, não diferem entre si ao nível de 5\% pelo teste de Ducan. Amostras: 20 ratas Wistar (5 ratas por grupo) \pm desvio padrão amostral da média. G1: recebeu dieta controle - OVX - continuou recebendo dieta controle. G2: recebeu dieta controle - OVX - passou a receber dieta yacon. G3: recebeu dieta yacon - OVX - passou a receber dieta controle. G4: recebeu dieta yacon - OVX continuou recebendo dieta yacon.

Seguindo as recomendações de Roberfroid, Gibson e Delzenne ${ }^{(17)}$ para cálculo do teor calórico das dietas, a dieta teste, do presente estudo, foi computada com 367,29 kcal/100 g, apresentando menor teor calórico que a dieta controle com 385,28 kcal/100 g. Esses valores calculados são menores, porém, proporcionais àqueles encontrados na análise de calorimetria, no qual demonstrou que essa mesma dieta teste também apresentou menor caloria $(407,5 \mathrm{kcal} / 100 \mathrm{~g})$ comparada à dieta controle $(442,9 \mathrm{kcal} / 100 \mathrm{~g})$.

Observa-se igualmente que os grupos que receberam dieta suplementada com PBY (produto à base de yacon), continuamente, apresentaram menor CEA e demonstram a capacidade de ganho de peso menos acelerado em função da ingestão da dieta contendo PBY. A seguir, encontram-se dispostos os IMCs (índices de massa corporal). 
Figura 2 - IMC de animais OVX que receberam dieta controle/dieta yacon (6\% FOS/inulina), durante 180 dias, e estão delineadas em diferentes condições experimentais.

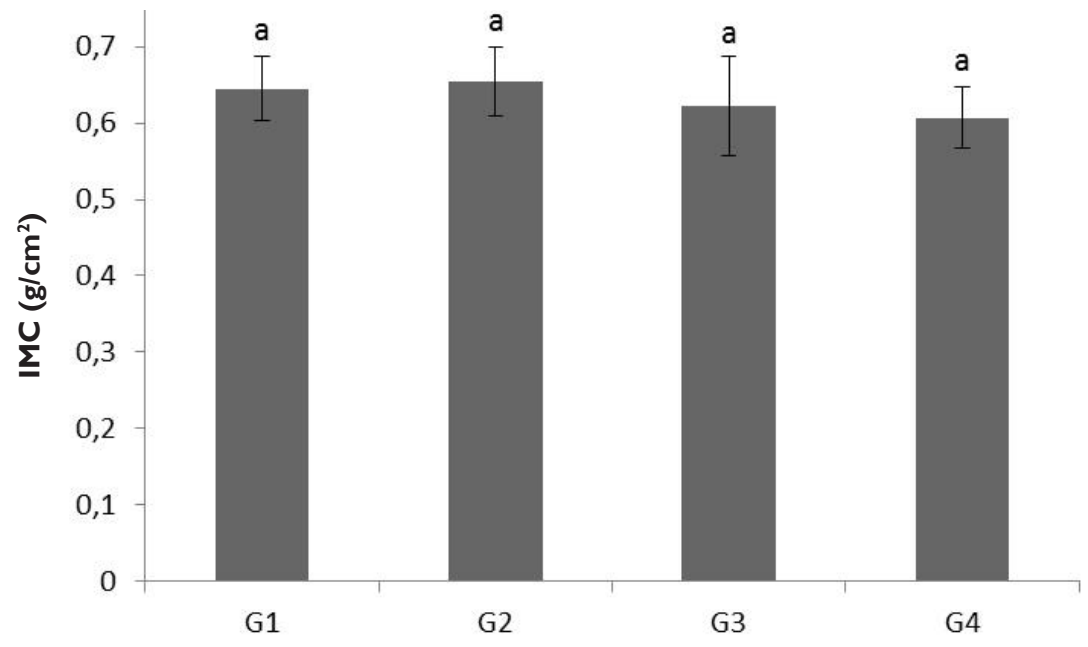

Fonte: Autoras

Legenda: Amostras: 20 ratas Wistar (5 ratas por grupo). G1: dieta controle - OVX - dieta controle. G2: dieta controle - OVX - dieta yacon. G3: dieta yacon - OVX - dieta controle. G4: dieta yacon - OVX - dieta yacon. Período antes da cirurgia (OVX) - 12 semanas. Período de recuperação (washout) 4 semanas. Período após a cirurgia $(\mathrm{OVX})-8$ semanas.

A queda no IMC no grupo G4 ( $\mathrm{p}=0,3822) \quad$ (simulação da menopausa), ao invés do acúmulo demonstra, em termos numéricos (sem diferença de gordura corporal e aumento do peso, demonstra significativa dos demais grupos), diminuição de queda do IMC. Abaixo, encontram-se dispostas as 6,25\% nesse índice. Esse grupo, que após a OVX circunferências das cinturas dos animais.

Figura 3 - Circunferência de animais OVX que receberam dieta controle/dieta yacon (6\% FOS/inulina), durante 180 dias, e estão delineadas em diferentes condições.

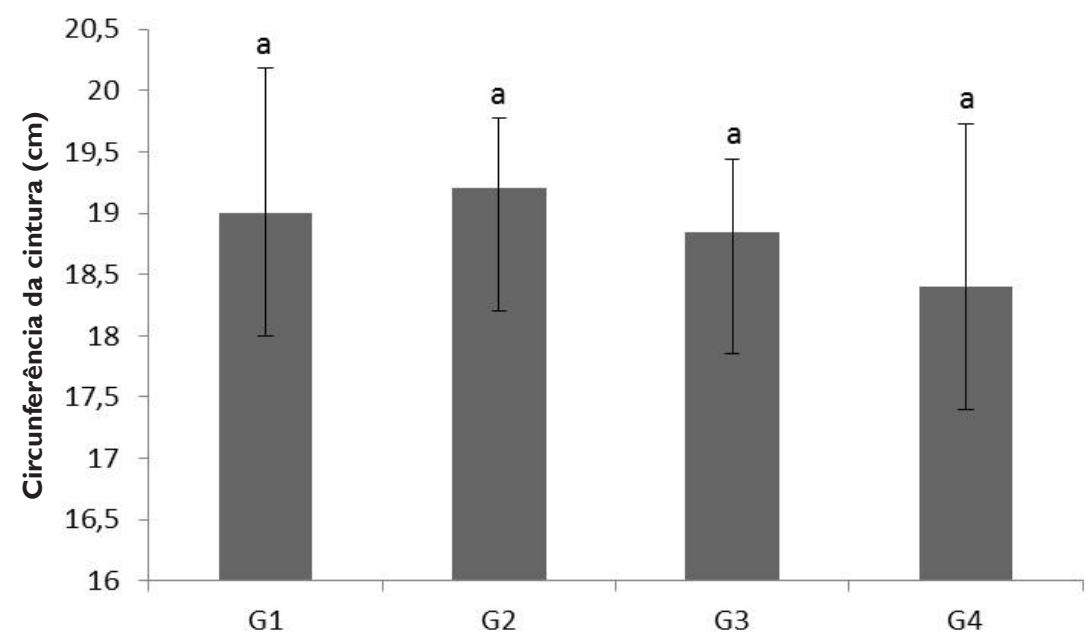

Fonte: Autoras

Legenda: Amostras: 20 ratas Wistar. G1: dieta controle - OVX - dieta controle. G2: dieta controle - OVX - dieta yacon. G3: dieta yacon - OVX dieta controle. G4: dieta yacon - OVX - dieta yacon. Período antes da cirurgia (OVX) - 12 semanas. Período de recuperação (washout) - 4 semanas. Período após a cirurgia $(\mathrm{OVX})-8$ semanas. 
No presente estudo, ocorre tendência ao aumento da gordura abdominal pós-cirurgia de ovariectomia; isso é possível ser verificado ao comparar com os dados de ratas celiotomizadas $\mathrm{p}<0,05$ (dados não mostrados). De acordo com os dados, observa-se uma menor circunferência no grupo $\mathrm{G} 4(\mathrm{p}=0,2173)$ apresentando decréscimo de 3,5\% para esse parâmetro, em função do uso da dieta suplementada com PBY, durante todo o experimento em relação ao grupo G1.

$\mathrm{Na}$ sequência, encontram-se dispostas as porcentagens de gordura corporal.

Figura 4 - Percentual de gordura corporal (\%GC) de animais OVX de ratas que receberam dieta controle/dieta yacon (6\% FOS/inulina), durante 180 dias, e estão delineadas em diferentes condições experimentais.

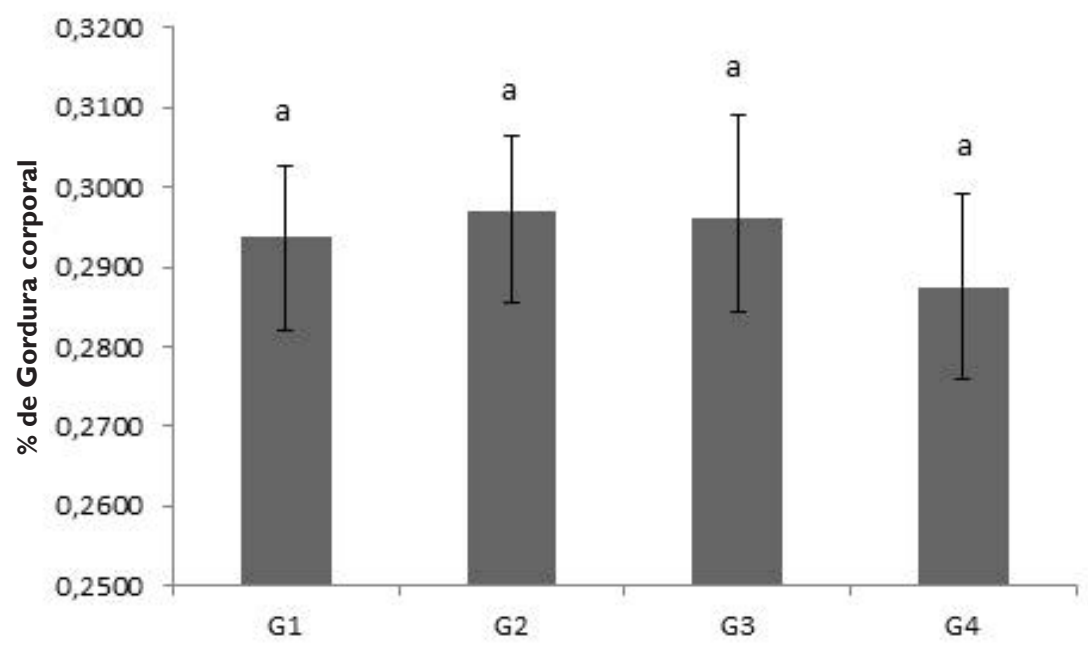

Fonte: Autoras

Legenda: Amostras: 20 ratas Wistar (5 ratas por grupo) \pm desvio padrão amostral da média. G1: dieta controle - OVX - dieta controle. G2: dieta controle - OVX - dieta yacon. G3: dieta yacon - OVX - dieta controle. G4: dieta yacon - OVX - dieta yacon. Período antes da cirurgia (OVX) 12 semanas. Período de recuperação (washout) - 4 semanas. Período após a cirurgia $(\mathrm{OVX})-8$ semanas.

O percentual de gordura corporal apresentado na Figura 4 demonstrou uma queda $(\mathrm{p}=0,3528)$ no grupo que recebeu dieta suplementada com PBY durante todo período experimental (G4), o que significa em termos numéricos uma queda de 2,14\% da gordura corporal, porém sem diferença significativa em relação aos outros grupos.

\section{Discussão}

O resultado de 9,24 g 100g-1, último resultado, foi inferior ao encontrado por Rodrigues et al. ${ }^{(18)}$ e Genta et al., ${ }^{(19)}$ que obtiveram 25,7 g 100g-1 e 44,2 g 100g-1 respectivamente. No presente estudo, um dos valores encontrados foi superior ao valor encontrado por Sant'Anna, ${ }^{(20)}$ a qual encontrou um teor de 14,54 g 100g-1. Estas variações de oligofrutanos podem ser decorrentes do período de cultivo e da colheita, tempo de armazenamento e das condições de estocagem. Essas variações devem ser levadas em consideração para elaboração do produto, o que sugere maior investigação do armazenamento e período de colheita do yacon para evitar degradação do FOS/inulina para que no produto elaborado (PBY) garanta-se os teores elevados de FOS/inulina.

Ao observar a Figura 2 (IMC), Figura 3 (circunferência cintura) e Figura 4 (\% de gordura corporal) não foi possível encontrar diferença significativa entre os grupos ao analisar esses 
parâmetros referidos, e sim uma tendência à queda. Ao passo que os animais que receberam dieta suplementada com PBY, durante todo o experimento (G4), apresentaram o menor ganho de peso $(\mathrm{p}<.0001)$, sugerindo que o hábito do consumo do produto apresenta potencial de diminuir risco da obesidade tendenciada pela hipoestrogenia estabelecida com a menopausa. Pode-se, portanto, sugerir que a inclusão do produto à base de yacon na dieta antes da menopausa, e a manutenção desse hábito alimentar durante essa fase, diminui o risco do estabelecimento da obesidade.

E mais, mesmo a dieta teste (suplementada com PBY) tendo sido mais consumida com aumento de consumo significativo $(p=0.0064)$ que a dieta controle, o ganho de peso do grupo de maior consumo foi significativamente menor $(\mathrm{p}<.0001)$. Nesse contexto, um grupo que merece destaque é o grupo G4, que correspondeu ao grupo de menor peso mesmo tendo consumindo mais dieta comparado ao grupo G1. Paula ${ }^{(21)}$ sugere que a grande presença de fibras na dieta contendo PBY, pode ter diminuído seu valor calórico, o que pode ter induzido ao maior consumo. Ainda assim, esse resultado é promissor, uma vez que um maior consumo não resultou em aumento proporcional no ganho de peso.

Em um primeiro instante, pode-se remeter a desaceleração no ganho de peso ao alto teor de fibras, como citado no estudo de Paula, ${ }^{(21)}$ a qual, consequentemente, diminui a densidade energética. Esse fato reforçaria a situação dos ratos ingerirem mais dieta teste e ganharem menos peso, resultado esse de grande relevância, pois determinaria a perda de peso, e, consequentemente, a diminuição das comorbidades relacionadas.

Ademais, mesmo a dieta teste (grupo G4) apresentando menor valor calórico, a quantidade ingerida resulta em ingestão de mais calorias, ou seja, ingerindo-se 18,4 g de dieta controle (G1) resulta em uma ingestão de aproximadamente 71 $\mathrm{kcal}$, ao passo que ingerindo $21,2 \mathrm{~g}$ de dieta teste (G4) resulta na ingestão de aproximadamente 78 kcal. Com esses dados, sugere-se que a relação estabelecida entre a maior ingestão de calorias e maior consumo da dieta, tendo como resultado um menor ganho de peso, pode estar ligado ao fato que as fibras diminuem a eficiência de absorção de lipídeos ao longo do intestino delgado, o que, consequentemente, diminui a assimilação calórica dos nutrientes. ${ }^{(22)}$ Acredita-se também que os ácidos orgânicos, em especial o butirato, agem na modulação do metabolismo de macronutrientes, mais especificamente de carboidrato e lipídeos, e agem na redução do ganho de peso, entretanto o mecanismo não está totalmente elucidado. ${ }^{(23)}$

Ocorreu um aumento na contagem de GLP-1 nos grupos que receberam PBY, em especial no grupo que recebeu PBY durante todo experimento (Tabela 1). De acordo com Drucker, ${ }^{(24)}$ o aumento de GLP-1 pode promover a saciedade, entretanto, paradoxalmente, ocorreu maior consumo de alimentos nos grupos que apresentaram maior contagem de GLP-1, no entanto, com diminuição de ganho de peso. Acredita-se que esse fenômeno pode reforçar a hipótese de Rolls, ${ }^{(2)}$ que a desaceleração no ganho de peso pode ser em decorrência da menor assimilação calórica de nutriente proporcionada por alimentos com alta ingestão de fibras.

Ainda em relação ao consumo alimentar apresentado, acredita-se que a melhor palatabilidade do PBY pode ter influenciado no aumento do consumo, que segundo Bion et al., ${ }^{(25)}$ a palatabilidade é um fator de grande influência na ingestão de dietas pelos animais de laboratório. Esse fato resultou em um menor consumo médio de dieta pelo grupo G1, que recebeu dieta controle (não suplementada com PBY), ao longo de todo período experimental, comparada com o grupo G4, do qual consumiu somente dieta teste durante todo experimento.

Observa-se, do mesmo modo, que os grupos que receberam dieta suplementada com PBY, continuamente, apresentaram menor CEA e demonstram a capacidade de ganho de peso menos acelerado em função da ingestão da dieta contendo PBY. O CEA analisa a capacidade dos animais perderem ou ganharem peso quando submetidos a diferentes dietas (análise global ou específica de nutrientes). Sendo assim, quando pensa-se que na menopausa, ou OVX em ratas, a tendência é um 
aumento da gordura corporal (GC) e ganho de peso localizado na região abdominal, essa diminuição de ganho de peso comparado aos animais do grupo controle pode ser considerada benéfica.

Sant'Anna, ${ }^{(20)}$ ao avaliar a adição de PBY na dieta e na modulação da constipação intestinal, e Rodrigues et al., ${ }^{(18)}$ ao avaliarem a modulação na absorção de cálcio por meio da suplementação da dieta com farinha de yacon, nas concentrações de $4 \%$ e $8 \%$, não encontraram diferença significativa no peso, no consumo alimentar e no coeficiente de eficiência alimentar em dieta suplementada com yacon. Entretanto, ambos os estudos foram realizados por 28 dias, ao passo que o presente estudo computou 180 dias. Esse fato pode ter influenciado nos resultados, visto que na presente pesquisa a suplementação da dieta com PBY foi realizada por um período longo, que representou $14 \%$ de toda a vida do animal, considerando que os ratos de laboratório vivem, em média, três anos. Extrapolando esse tempo para os humanos, representaria o consumo do PBY por um período de 15 anos, ao passo que nos trabalhos citados anteriormente representou apenas $2,6 \%$ da vida do animal, e apenas dois anos e três meses de vida do ser humano. ${ }^{(26)}$

Esses resultados são de extrema importância como um indicador para estudos clínicos, uma vez que um alimento funcional agindo na prevenção, ou até mesmo na modulação da tendência à obesidade, é fundamental, principalmente pela possibilidade de atuação como adjunto aos tratamentos já existentes, à reeducação alimentar e à prática de exercícios físicos. A modulação da obesidade pode diminuir o risco de DM2, ${ }^{(27,28)}$ $\operatorname{HAS}^{(27,28)}$ e dislipidemias, ${ }^{(27,28)}$ distúrbios comuns na menopausa. A dislipidemia, por sua vez, é um importante fator de risco para o desenvolvimento de doenças coronarianas, com consequente redução na qualidade de vida. ${ }^{(29)}$

O grupo G4, que após a OVX (simulação da menopausa), ao invés do acúmulo de gordura corporal e aumento do peso, demonstra queda do IMC. Esse resultado reforça a importância do uso do produto à base de yacon na modulação da obesidade, em especial na menopausa em virtude da sua tendência ao acúmulo de gordura.

De acordo com Duarte e Reis, ${ }^{(30)}$ em idosos o IMC tende a ter um acréscimo em torno de 1,5 $\mathrm{kg} / \mathrm{m}^{2}$ em homens e $2,5 \mathrm{~kg} / \mathrm{m}^{2}$ em mulheres. Em estudos realizados em países desenvolvidos, com indivíduos adultos, de ambos os sexos, foram verificadas correlações entre IMC e indicadores murinométricos de gordura não visceral e de gordura abdominal, bem como relação direta entre IMC com a massa de gordura corporal total. ${ }^{(31)}$ Segundo Amann et al., ${ }^{(32)}$ o IMC está associado à mortalidade cardiovascular, tanto em homens quanto em mulheres. De forma similar, Novelli et $a l .^{(13)}$ defendem que o IMC também pode ser usado como um instrumento de avaliação da gordura corporal em ratos, bem como um indicador de alterações lipidêmicas. Então, alternativas que resultem em uma diminuição desse parâmetro são de grande valia, pois podem prevenir consequências graves em longo prazo.

No presente estudo, ocorre tendência ao aumento da gordura abdominal pós-cirurgia de ovariectomia (OVX); isso é possível ser verificado ao comparar com os dados de ratas celiotomizadas $\mathrm{p}<0,05$ (dados não mostrados). Esses resultados corroboram os resultados de Franco, ${ }^{(33)}$ o qual, ao avaliar o efeito da cafeína e exercício físico em ratas ovariectomizadas, observou que após a retirada do ovário ocorre um aumento significativo da circunferência abdominal.

Vale lembrar que a OVX simula o que ocorre com mulheres na menopausa, do qual determina a deposição de gordura, ${ }^{(33)}$ e, por isso, uma redução nesse parâmetro evidencia a importância da ingestão de PBY, e, mais especificamente, do uso de forma contínua para potencializar os resultados. Essa hipótese é reforçada com a diminuição de $3,5 \%$ de circunferência abdominal do grupo G4 comparada ao grupo G1.

Importante ressaltar que, conforme o estudo International Day for the Evaluation of Abdominal Obesity (IDEA), ${ }^{(34)}$ o tecido adiposo na região abdominal é um excelente indicativo para risco de doenças crônicas, entre elas a hipertensão arterial e 
a diabetes mellitus tipo 2, o que resulta em menor qualidade de vida e maior gasto governamental. $\mathrm{O}$ presente estudo promovendo essa diminuição torna-se de grande relevância, pois diminui o risco dessas enfermidades.

O percentual de gordura corporal (\%GC) do estudo demonstrou uma queda $(\mathrm{p}=0,3528)$ no grupo que recebeu dieta suplementada com PBY durante todo período experimental (G4), o que significa em termos numéricos uma queda de $2,14 \%$ da gordura corporal demonstrando novamente a importância do efeito do uso do PBY em uso contínuo. Esse resultado de modulação da gordura corporal é muito relevante, pois com a menopausa ocorre a maior deposição de gordura, resultando em maior ganho de peso, e essa deposição de gordura na região abdominal resulta no maior risco de doença cardiovascular, menor qualidade de vida e maior gasto governamental.

Segundo Bernardis e Patterson, ${ }^{(14)} \mathrm{o}$ índice de Lee maior que 0,3 pode ser usado como indicador do excesso de gordura corporal. Desse modo, ao observar a Figura 4 verifica-se que os percentuais de gordura corporal de todos os grupos estão dentro do recomendado; entretanto, no grupo G4 os índices são ligeiramente menores, o que pode ser um fator benéfico para diminuição desse índice, caso o mesmo esteja aumentado, ou seja, segundo Bernardis e Patterson, ${ }^{(14)}$ um $\% \mathrm{GC}>0,3$.

\section{Conclusões}

A diferença estatisticamente significativa encontrada em relação ao ganho de peso no grupo que recebeu PBY durante todo o experimento demonstrou o potencial desse alimento na modulação da obesidade, sugerindo-se que o hábito de consumo do yacon continuamente tem potencial para diminuir os riscos de estabelecimento dessa comorbidade e, portanto, melhoria da qualidade de vida. Da mesma maneira que o aumento significativo de GLP-1 nesse grupo supracitado também está intimamente ligado ao controle de peso e à modulação da diabetes mellitus tipo 2, ambas comorbidades comuns da menopausa.
No entanto, novos estudos devem ser estimulados para melhor entendimento dos mecanismos envolvidos, para estabelecer qual a dose necessária de consumo de yacon e qual o melhor tempo de uso, sobretudo em ensaios clínicos.

\section{Referências}

1 Murador DC, Rosso VV. Efeito do preparo da couve (Brassica oleracea L.) na composição quali e quantitativa dos compostos bioativos. Nutrire. 2013;38(supl.):197.

2 Ricarte D, Júlio BLA, Zocateli GAF, Barreto RLF, Guimarães M, Ferreira RS, et al. Análise sensorial de preparações com batata yacon: revisão sistemática. HU Rev. [Internet]. 2020 [citado 2020 jun 17];45(4):431-40. Disponível em: https://periodicos.ufjf.br/index.php/ hurevista/article/view/28419

3 Gusso AP, Mattanna P, Richards N. Yacon: benefícios à saúde e aplicações tecnológicas. Ciência Rural. 2015 Maio;45(5):912-9. doi: 10.1590/0103-8478cr20140963.

4 Dias PC, Henriques P, Anjos LA, Burlandy L. Obesidade e políticas públicas: concepções e estratégias adotadas pelo governo brasileiro. Cad. Saúde Pública. 2017 jul;33(7):e00006016. doi: 10.1590/0102-311x00006016.

5 Gonçalves JTT, Silveira MF, Campos MCC, Costa LHR. Sobrepeso e obesidade e fatores associados ao climatério. Ciênc. Saúde Colet. 2016 Abr;21(4):1145-56. doi: 10.1590/141381232015214.16552015 .

6 Ferreira APS, Szwarcwald CL, Damacena GN. Prevalência e fatores associados da obesidade na população brasileira: estudo com dados aferidos da Pesquisa Nacional de Saúde, 2013. Rev. Bras. Epidemiol. 2019 Abr; 22:e190024. doi: 10.1590/1980-549720190024.

7 Associação Brasileira para o Estudo da Obesidade e da Síndrome Metabólica (ABESO/ SBEM). Atualização das diretrizes oficiais para tratamento farmacológico da obesidade e sobrepeso [Internet]. 2010. [citado 2020 jun 17]. Disponível em: https://abeso.org.br/wp-content/ uploads/2019/12/Atualizacao-das-Diretrizes.pdf 
8 Grancieri M, Machado PA, Oliveira DF, Marcon LN, Tostes MGV, Costa NMB, et al. Efeito da farinha de yacon (Smallanthus sonchifolius) na resposta imunológica intestinal no câncer colorretal. Braspen J. 2016 Out; 31(4):35-9.

9 Association of Official Analytical Chemists (AOAC). Official methods of analysis. $18^{\text {th }} \mathrm{ed}$. Washinghton, DC; 1998.

10 Reeves PG, Nielsen FH, Fahey GC Jr. AIN93 purified diets for laboratory rodents: final report of the American Institute of Nutrition ad hoc writing committee on the reformulation of the AIN-76A rodent diet. J Nutr. 1993 Nov;123(11):1939-51. doi: 10.1093/jn/123.11. 1939.

11 Mezadri TJ, Tomáz VA, Amaral VLL. Animais de laboratório: cuidados na iniciação experimental. Florianópolis: Ed. UFSC; 2004.

12 Jaime APG, Anbinder AL, Lima AP, Prado FA, Balducci I, Rocha RF. Influência da administração local de alendronato sódico no reparo ósseo em calvária de ratas ovariectomizadas. Cienc. Odontol. Bras. 2005 Abr; 8 (2):70-9. doi: 10.14295/bds.2005. v8i2.391.

13 Novelli EL, Diniz YS, Galhardi CM, Ebaid GM, Rodrigues HG, Mani F, et al. Anthropometrical parameter and markers of obesity in rats. Lab Anim. $2007 \mathrm{Fev}$;41(1):1119. doi: $10.1258 / 002367707779399518$.

14 Bernardis LL, Patterson BD. Correlation between 'Lee index' and carcass fat content in weanling and adult female rats with hypothalamic lesions. J Endocrinol. 1968 Apr; 40(4):527-8. doi: 10.1677/joe.0.0400527.

15 Sternberger LA, Hardy PH Jr, Cuculis JJ, Meyer HG. The unlabeled antibody enzyme method of immunohistochemistry: preparation and properties of soluble antigen-antibody complex (horseradish peroxidase-antihorseradish peroxidase) and its use in identification of spirochetes. J Histochem Cytochem. 1970 May;18(5):315-33. doi: 10.1177/18.5.315.

16 Bancroft JD, Stevens A. Theory and practice of histological techniques. Nova York: Churchill livingstone; 1996.
17 Roberfroid M, Gibson GR, Delzenne N. The biochemistry of oligofructose, a nodigestible fiber: an approach to calculate its caloric value. Nutr Rev. 1993 May;51(5):137-46. doi: 10.1111/j.1753-4887.1993.tb03090.x.

18 Rodrigues FCR, Castro ASB, Rodrigues VC, Fernandes SA, Fontes EAF, Oliveira $\mathrm{TT}$, et al. Yacon Flour and Bifidobacterium longum modulate bone. Health in Rats. J Med Food 2012 Apr;15(7):664-70. doi: 10.1089/ jmf.2011.0296.

19 Genta S, Cabrera W, Habiba N, Ponsb J, Carilloc IM, Graud A, Sánchez S. Yacon syrup: beneficial effects on obesity and insulin resistance in humans. Clin Nutr. 2009 Apr; 28 (2): 182-7. doi: 10.1016/ j.clnu.2009.01.013.

20 Sant'Anna MSL. Efeito de um produto a base de yacon (Smallanthus sonchifolius) na modulação da constipação intestina. [tese]. Viçosa: Universidade Federal de Viçosa-UFV; 2013.

21 Paula HAA. Efeito de um produto à base de yacon (Smallanthus sonchifolius) na modulação de indicadores da saúde óssea em ratas Wistar [tese]. Viçosa: Universidade Federal de Viçosa - UFV; 2013.

22 Rolls BJ. The role of energy density in the overconsumption of fat. J Nutr. 2000 Feb;130(2):268S-71S. doi: 10.1093/jn/130.2. $268 \mathrm{~S}$.

23 Maham LK, Escott-Stump S. Alimentos, nutrição e dietoterapia. 11a ed. São Paulo: Roca; 2005.

24 Drucker DJ. Minireview: the glucagon-like peptides. Endocrinology. 2001 Feb; 142(2): 521-7. doi: 10.1210/endo.142.2.7983.

25 Bion FM, Pessoa DCNP, Lapa MAG, Carvalho MJ. Uso de uma multimistura como suplementação alimentar: estudo em ratos. Arq Lat. Amer. Nutr. 1997;47(3):242-7.

26 Andreollo NA, Santos EF, Araújo MR, Lopes LR. Idade dos ratos versus idade humana: qual é a relação? Arq Bras Cir Dig. 2012 Mar;25:(1):49-51. doi: 10.1590/S0102-672020 12000100011 . 
27 Associação Brasileira para o Estudo da Obesidade e da Síndrome Metabólica (ABESO/ SBEM). Diretrizes Brasileiras de Obesidade da Associação Brasileira para o Estudo da Obesidade e da Síndrome Metabólica. São Paulo: Mazza; 2009.

28 ADA - American Diabetes Association. Standars of medical care in diabetes. Diabetes Care. 2010, 33(Suppl 1):S11-S61.

29 Lima AN. Fatores Associados ao Excesso de Peso entre os Usuários do Serviço de Promoção à Saúde: Academia da Cidade do Distrito Sanitário Leste de Belo Horizonte Minas Gerais [dissertação]. Belo Horizonte (MG): Universidade Federal de Minas Gerais (UFMG); 2009.

30 Duarte SFP, Reis LAR. Obesidade: uma visão multidisciplinar. Curitiba: CRV; 2012.

31 Navarro AM, Marchini JS. Uso de medidas antropométricas para estimar gordura corporal em adultos. Nutrire. 200019 (1):31-47.

32 Amann VR, Santos LP, Gigante DP. Associação entre excesso de peso e obesidade e mortalidade em capitais brasileiras e províncias argentinas. Cad. Saúde Pública. 2019 Nov; 35(12): e00192518. doi: 10.1590/0102-311x00192518.

33 Franco FSC. Efeitos da ovariectomia, ingestão de cafeína e exercício aeróbio associados à adequação ou não de cálcio alimentar na composição corporal, no tecido ósseo e no balanço de cálcio em ratas. [tese]. Viçosa: Universidade Federal de Viçosa (UFV); 2009.

34 Balkau B, Deanfiels JE, Després JP, Bassand JP, Fox KAA, Smith Jr SC, et al. International Day for the Evaluation of Abdominal Obesity (IDEA): a study of waist circumference, cardiovascular disease, and diabetes mellitus in 168,000 primary care patients in 63 countries. Circulation. 2007 Out;116(17):1942-51. doi: 10.1161 / CIRCULATIONAHA.106.676379. 